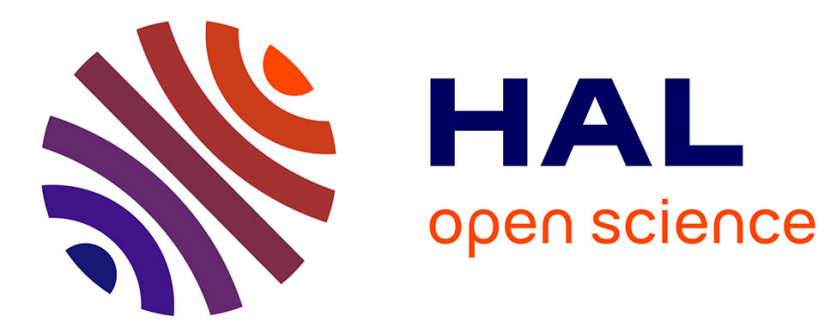

\title{
La question du consentement sexuel: entre liberté individuelle et dignité humaine
}

\author{
David Simard
}

\section{To cite this version:}

David Simard. La question du consentement sexuel: entre liberté individuelle et dignité humaine. Sexologies, 2015, 24 (3), 10.1016/j.sexol.2015.05.003 . hal-01224567

\section{HAL Id: hal-01224567 \\ https://hal.science/hal-01224567}

Submitted on 22 Aug 2016

HAL is a multi-disciplinary open access archive for the deposit and dissemination of scientific research documents, whether they are published or not. The documents may come from teaching and research institutions in France or abroad, or from public or private research centers.
L'archive ouverte pluridisciplinaire HAL, est destinée au dépôt et à la diffusion de documents scientifiques de niveau recherche, publiés ou non, émanant des établissements d'enseignement et de recherche français ou étrangers, des laboratoires publics ou privés.

\section{(1) (1) $\$$}

Distributed under a Creative Commons Attribution - NonCommercial - NoDerivatives| 4.0 


\title{
La question du consentement sexuel : entre liberté individuelle et dignité humaine
}

\author{
David Simard
}

Sexologies (2015) 24, 140-148

Mots clés : Consentement sexuel ; Désir ; Volonté ;

Sadomasochisme ; Prostitution ; Travail sexuel ; Dignité humaine

Résumé : En matière sexuelle, le consentement est une notion devenue primordiale. Elle permet sur le plan juridique, notamment, de distinguer ce qui ne relève pas du viol et ce qui en relève. C'est toutefois une notion aux contours mal définis, qui donne lieu à des controverses, particulièrement sur la question de la prostitution et sur celle du sadomasochisme pour ce qui concerne l'éthique de la sexualité. Cet article a pour objectif de tenter d'éclairer les termes du débat. II interroge d'abord les fondements du consentement sexuel, en analysant les différences ou les confusions conceptuelles entre le désir et la volonté, tout d'abord à partir de la lecture des philosophes du XVIle siècle René Descartes et Baruch Spinoza, qui s'opposent sur la place du désir relativement à la raison chez l'être humain, et ensuite du psychanalyste Sigmund Freud, qui introduit l'idée de désir inconscient. L'article montre ensuite comment l'impossibilité d'une liberté absolue, et donc d'un consentement dégagé de toute contrainte, peut conduire certaines organisations féministes à mettre en question le consentement individuel même clairement formulé, et à lui opposer la notion de dignité humaine en un sens transcendant, inspirée du philosophe allemand du XVIIIe siècle Emmanuel Kant. Cette notion est interrogée et étudiée dans les cas de la prostitution et du sadomasochisme. L'analyse conceptuelle conduit à mettre en évidence le caractère métaphysique tant de l'approche individualiste du consentement que de l'approche d'inspiration kantienne de la dignité humaine.

\section{Introduction}

Une des notions centrales dans le domaine de l'éthique de la sexualité est celle de consentement. C'est elle notamment qui est convoquée dans les rapports sexuels pour distinguer entre ce qui relève de l'agression sexuelle ou non. Or cette notion ne va pas de soi, avec d'un côté, une conception du consentement qui le fonde sur la volonté ou qui le dissocie du désir (Caouette, 2015), et d'un autre côté, une conception qui le fonde sur le désir ou qui considère 
qu'un consentement à des rapports sexuels non désirés est un consentement à une effraction source de traumatisme (Martine, 2013). Si ces deux vocables, volonté et désir, sont souvent confondus et considérés comme interchangeables dans le langage courant, il convient d'identifier les distinctions qui permettent de nommer deux processus différents qui fondent de manières différentes, et qui peuvent même parfois s'opposer, le rapport à l'acte ou au comportement. Ainsi peut-on désirer quelque chose mais ne pas le vouloir, ce qui arrive communément dans le domaine sexuel (on peut par exemple être en couple et désirer une tierce personne, mais ne pas vouloir suivre ce désir par fidélité à son conjoint). On peut par ailleurs vouloir un rapport sexuel, mais pour d'autres motifs que le désir, ce qui arrive là aussi communément (par recherche de valorisation narcissique, pour calmer une angoisse, pour de l'argent, etc.).

Se pose alors la question de la valeur du consentement, lorsque ce à quoi il est consenti paraît contrevenir à des valeurs morales socialement tenues pour importantes. La notion de dignité humaine est ainsi convoquée par certains courants féministes pour rejeter l'idée qu'est recevable le consentement à des rapports sexuels prostitutionnels, désignés comme du travail sexuel comme par exemple en France par le Syndicat du travail sexuel (STRASS), ou relevant du sadomasochisme notamment. Le point de référence n'est alors plus l'individu, qu'il s'agisse de son désir ou de sa volonté, mais un principe supérieur auquel l'individu devrait se conformer.

Ce sont ces deux problématiques, celle de la fondation du consentement sur le désir ou la volonté, d'une part, et celle de la reconnaissance du consentement par un principe de transcendance, d'autre part, que les lignes qui suivent vont mettre en lumière. L'enjeu est celui de l'autonomie de la personne et de sa liberté sexuelle dans un contexte inter-relationnel.

\section{Quelques données statistiques sur les agressions sexuelles, la prostitution et le sadomasochisme}

\section{Les agressions sexuelles}

Les rapports sexuels non consentis sont des agressions sexuelles. Cette affirmation peut paraître aller de soi, mais les discours que l'on a pu entendre à l'occasion, par exemple, des affaires impliquant I'homme politique français Dominique Strauss-Kahn contre la femme de chambre Nafissatou Diallo en 2011, et dans le cadre du procès pour proxénétisme aggravé en réunion dit procès de "l'affaire du Carlton » en 2015, ont mis en évidence le présupposé que, dès lors qu'une femme exerce la prostitution, ou dès lors qu'elle est libertine, il ne peut y avoir de viol, parce qu'il n'y a plus à s'enquérir de son consentement compte tenu de son mode d'inscription dans le champ sexuel (Simard,2011). 
Selon l'Organisation mondiale de la santé (OMS), au niveau mondial, $35 \%$ des femmes indiquent avoir été exposées à des violences sexuelles de la part de leur partenaire intime ou de quelqu'un d'autre à un moment de leur vie (OMS, 2014). Aux États-Unis, $18,3 \%$ des femmes et $1,4 \%$ des hommes déclarent avoir été violés au cours de leur vie, et $44,6 \%$ des femmes ainsi que $22,2 \%$ des hommes déclarent avoir subi d'autres violences sexuelles hors pénétration (Black et al., 2011). En France, l'enquête "Contexte de la sexualité en France » de 2006 permet de calculer que 20,4\% des femmes et $6,8 \%$ des hommes de 18 à 69 ans se déclarent victimes d'agression sexuelle physique, comprenant les attouchements, les tentatives de rapports forcés et les rapports forcés. Au total, 15,5\% des femmes et $4,8 \%$ des hommes déclarent par ailleurs avoir subi des conversations à caractère pornographique (Bajos et Bozon, 2008).

\section{Les chiffres de la prostitution}

Avec toute une partie de la prostitution qui est dominée par des réseaux criminels et le trafic d'êtres humains, et compte tenu d'enjeux idéologiques importants qui viennent parasiter les principes de la recherche scientifique (Weitzer, 2005), le nombre de personnes exerçant une activité de prostitution ou de travail sexuel dans le monde est difficile voire impossible à établir. En France, la fondation Scelles a publié, en 2012, la première édition de son rapport mondial sur l'exploitation sexuelle qui avançait le chiffre de 40 à 42 millions de personnes pratiquant la prostitution dans le monde (Charpenel, 2012). Pour la France, le rapport d'information sur le renforcement de la lutte contre le système prostitutionnel, déposé à l'Assemblée nationale en 2013 (Olivier, 2013), se réfère au rapport annuel de2010 de l'Office central pour la répression de la traite des êtres humains (OCRTEH) et avance une fourchette comprise entre 20000 et 40000 personnes se prostituant. Ces chiffres sont notamment contestés par le Syndicat du travail sexuel (STRASS), pour lequel le rapport d'information de Maud Olivier, qui propose la pénalisation des clients de la prostitution, recourt à des arguments biaisés (Schaffauser, 2014).

\section{Les pratiques et fantasmes sadomasochistes}

Les chiffres résultant d'enquêtes en population générale sur les adeptes des pratiques sexuelles sadomasochistes sont peu nombreux. On peut citer une étude menée en Australie qui rapporte que $1,8 \%$ des personnes sexuellement actives (2,2 \% d'hommes et $1,3 \%$ de femmes) ont déclaré avoir eu des pratiques relevant du bondage et de la discipline, de la domination et de la soumission et du sadomasochisme (BDSM) l'année précédent l'enquête (Richters et al., 2008). En France, on trouve des données statistiques dans l'enquête, maintenant ancienne datant de 1993 et coordonnée par I'Inserm, sur l'analyse des comportements sexuels, non sur les pratiques BDSM, mais sur les fantasmes qui y ont trait. Selon cette 
enquête, $1,3 \%$ des hommes et $0,8 \%$ des femmes avaient souvent fantasmé de punir et blesser un partenaire, tandis que 1,8\% des hommes et $1,3 \%$ des femmes avaient souvent fantasmé avoir des rapports sexuels avec un partenaire attaché ou qui ne peut bouger. Par ailleurs, $1,2 \%$ des hommes et $0,5 \%$ des femmes avaient déclaré fantasmer souvent d'être puni ou blessé, et 0,7 \% des hommes et $1,5 \%$ des femmes d'avoir des rapports sexuels en étant attaché ou sans pouvoir bouger (Bajos et al., 1998).

\section{Le consentement, affaire de désir ou de volonté ?}

Ces données statistiques portent sur les rapports sexuels non consentis et les pratiques pour lesquelles nous allons étudier la valeur du consentement. Or la question du fondement du consentement renvoie à la conception que l'on se fait de l'être humain et des modalités d'interaction entre les personnes. Savoir si le consentement doit reposer sur la volonté ou sur le désir pose en effet la question de la place respective du rationnel et de l'irrationnel, du conscient et de l'inconscient dans les comportements humains. Cela pose ainsi la question de la construction du choix et du processus de décision, en rapport avec la dimension intime de l'individu, ce qui paraît le plus privé, a fortiori dans le domaine de la sexualité, mais toujours dans un contexte de relation à autrui.

Désir et volonté sont souvent confondus. Ils ne renvoient pourtant pas à la même chose, dans la mesure où ils ne se construisent pas de la même manière, même s'il serait artificiel de les considérer dans un rapport d'hermétisme total l'un à l'autre.

En un sens très général, le désir est un élan qui porte vers quelque chose. On pourrait alors y inclure la volonté, mais aussi bien le besoin, l'envie, le souhait, l'inclination, etc. Toutefois, un tel amalgame ne permet pas de penser les situations concrètes et de départager ce qui fait consentement ou non. Par exemple, l'association féministe française "Osez le féminisme ! " (OLF) associe le consentement et le désir. À propos du travail sexuel, cette association affirme notamment dans l'un de ses documents destiné aux députés afin de les inciter à voter en faveur de la proposition de résolution abolitionniste $n^{\circ} 3522$ présentée en séance en décembre 2011, que " si le consentement des femmes est conditionné par le besoin d'argent, il est biaisé ». Et d'ajouter plus loin : " on ne peut concevoir d'égalité entre les femmes et les hommes en dehors de toute réciprocité de désir et de plaisir, du libre choix de ses partenaires et de ses pratiques sexuelles » (OLF, 2011). C'est donc que, du point de vue de cette association féministe, le désir est une condition sine qua non du consentement auquel on puisse accorder une véritable valeur.

Le désir comme mouvement de la volonté commandée par la raison : l'approche cartésienne 
Mais en quoi le désir diffère-t-il de la volonté ? Dans la troisième maxime de sa morale par provision, Descartes préconisait de changer ses désirs plutôt que l'ordre du monde (Descartes, 1953a). Or ainsi que le philosophe français le développe, cette maxime repose sur l'idée que c'est la volonté qui désire, d'une part, et que, d'autre part, la volonté n'est portée à désirer que les choses que lui présente l'entendement, c'est-à-dire la raison. Changer ses désirs est donc possible parce que le désir est la volonté qui est elle-même sous l'empire de la raison, qui l'éclaire. Plus précisément, la raison détermine ce qui est accessible, et le présente à la volonté qui désire alors ce qui est accessible. Dans le cadre d'une telle articulation entre désir, volonté et raison (ou entendement), le consentement reposerait alors sur la volonté éclairée par la raison et consisterait en la détermination même du désir quant à son objet par une élaboration rationnelle. Descartes précise d'ailleurs dans Les passions de l'âme, alors qu'il parle de l'amour et de la haine, que le mot "volonté " désigne le " consentement par lequel on se considère dès à présent comme joint avec ce qu'on aime ", introduisant au passage une nuance entre la volonté et le désir, la première se rapportant au présent, le second à l'avenir (Descartes, 1953c).

\section{Le désir antérieur à la raison : la conception spinozienne}

Cette approche du désir comme finalement précédé par la raison sera remise en question, d'une part, par un quasi-contemporain de Descartes, à savoir Spinoza, et d'autre part, par le fondateur de la psychanalyse: Freud. Pour Spinoza, l'être humain est fondamentalement un être désirant. Dans la troisième partie de son Éthique, alors qu'il définit les affections, il donne la définition suivante du désir : "Le Désir est l'essence même de l'homme en tant qu'elle est conçue comme déterminée à faire quelque chose par une affection quelconque donnée en elle " (Spinoza, 1965).Cette primauté du désir chez l'être humain procède de ce qu'il appartient à l'essence de toute chose de chercher à persévérer dans son être. Le désir présente cette particularité qu'il n'est pas seulement l'effort pour persévérer dans son être, mais qu'il est en plus la conscience de cet effort (Spinoza, 1965).

Dès lors, le désir est antérieur à la raison, et si elle peut tenter d'ordonner les désirs selon une hiérarchie reposant sur l'accroissement ou la diminution de la perfection de l'esprit, ce n'est qu'après coup, et en procédant elle-même de l'effort pour persévérer dans l'être, mais sous la forme d'idées plutôt que d'affects. Contrairement à Descartes donc, il n'est pas pour Spinoza dans le pouvoir de la raison de changer les désirs. Ainsi, la raison n'a pas le pouvoir de choisir, après délibération, l'objet du désir, sur la base de ce qui sera jugé par elle comme bon ou vrai, accessible et dépendant de soi ; au contraire : " nous jugeons qu'une chose est bonne parce que nous nous efforçons vers elle, la voulons, appétons 
et désirons " (Spinoza, 1965). Le désir comme effort relevant de l'essence humaine précède ainsi tout objet à désirer, et les objets qu'il se donne relèvent plus des circonstances que d'un choix raisonné qui nous indiquerait comme désirable tel ou tel objet.

Observons qu'ici, vouloir et désirer se trouvent insérés dans une même série. Cela ne veut pas dire qu'ils signifient exactement la même chose, mais tous deux sont des modalités de la persévérance dans l'être. Ils diffèrent en ce que la volonté est une modalité de la persévérance dans l'être qui ne se rapporte qu'à l'esprit, tandis que le désir se rapporte à la fois à l'esprit et au corps. Ils ont par ailleurs en commun de ne pas nécessairement être placés sous l'empire de la raison. Par rapport à l'approche cartésienne, cela pose un problème quant à la manière dont on peut fonder le consentement : la volonté ou le désir ne peuvent plus être considérés comme le fruit d'un choix. Partant, si consentir c'est choisir librement, ni le désir ni la volonté ne peuvent être au fondement du consentement. Au contraire pour Spinoza, loin d'être le pouvoir d'agir selon son bon plaisir, la liberté réside chez " celui dont l'âme est saine et qui ne prend d'autre guide que la raison » (Spinoza, 1842).

\section{Le désir antérieur à la raison : l’approche freudienne}

Freud ira encore plus loin que Spinoza dans la prééminence du désir et le fait qu'il échappe à la raison quant à sa réalité et les objets sur lesquels il se porte. La partition désir/raison prend chez lui la forme d'une partition entre inconscient et conscient. Là où Spinoza définissait le désir comme l'effort pour persévérer dans son être accompagné de conscience, le psychanalyste le loge du côté de l'inconscient. II se rapproche d'une conception plus platonicienne que spinoziste du désir en le rapportant au manque, alors que Spinoza, inspirant plus tard Deleuze, en fait une force d'autoaffirmation qui se vit dans la joie : persévérer dans son existence n'est pas passer à l'existence à partir d'une situation de manque à être.

Freud, donc, conçoit le désir comme la recherche d'une satisfaction vécue antérieurement, associée à une représentation, et dont la force est telle que cette recherche conditionne la manière dont I'individu construit ses relations avec autrui. Mais la recherche de satisfaction peut entrer en contradiction avec d'autres critères, qui peuvent faire peser un interdit opposé à la satisfaction. Le plaisir qui serait obtenu par la satisfaction recherchée provoquerait du déplaisir par ailleurs, et même un déplaisir plus grand que ne le serait le plaisir. Dans ce cas, ainsi que Freud l'a observé par l'expérience de la clinique des névroses dans la pratique psychanalytique, un mécanisme de défense de refoulement peut être mis en œuvre. Or celui-ci consiste à tenir à l'écart de la conscience la représentation associée à la pulsion, ou plus exactement son substitut psychique qui peut prendre la forme d'une représentation («représentant-représentation», qui traduit 
"Vorstellungsrepräsentanz ») (Freud, 1968). Par exemple, une tendance hostile envers une personne aimée qui procurerait un plaisir sadique se voit refoulée parce que son accomplissement conduirait à un déplaisir plus grand (la perte de la personne aimée, une culpabilité envahissante).

Ainsi, le désir peut être interdit d'accès à la conscience, et même les désirs les plus puissants sont inconscients. On peut dire ici que si l'on considère la volonté comme ce qui résulte d'un choix conscient, raisonné ou non, celle-ci est susceptible de se situer en opposition avec le désir en tant qu'il est refoulé parce que, pour diverses raisons, pas acceptable. Pour autant, cela ne signifie pas que le désir, ou la pulsion qui le précède dans la conception freudienne de l'articulation de l'organique et du psychique, a été rendu inactif. Autrement dit, le désir même refoulé est agissant, et trouve des voies de réalisation ou de manifestation détournées et plus ou moins déguisées, comme dans les rêves, les fantasmes, les lapsus, les maladresses et autres actes manqués. II paraît dans de telles conditions hasardeux, dès lors, de considérer que le consentement doit avoir pour fondement le désir, c'est-à-dire finalement quelque chose dont la personne peut ne rien vouloir savoir.

La volonté, dès lors qu'elle se situe du côté du conscient, paraît plus indiquée. Cela ne signifie pas qu'elle $n^{\prime}$ ait rien à faire avec le désir inconscient, c'est-à-dire qu'il n'y ait rien dans ce qui est voulu qui n'émane de ce désir. Mais à défaut de pouvoir atteindre, comme le croyait notamment Descartes avec sa conception du " cogito ", à la transparence parfaite de soi-même, il semble difficile de trouver un meilleur fondement au consentement, qui ne peut relever d'une absolue liberté, soit d'une liberté qui consisterait en l'absence de tout déterminisme et de toute contrainte intérieurs comme extérieurs.

\section{Le consentement individuel mis en question}

C'est sans doute cette impossibilité d'une liberté absolue de l'individu qui conduit certaines organisations féministes, comme le Mouvement du nid en France, à se montrer critiques vis-à-vis du consentement. Comme le rappelait dans son dossier de presse le collectif « Abolition 2012 " regroupant cinquante-cinq associations féministes françaises, dont le Mouvement du nid, et dénonçant la prostitution comme une violence en soi, en se référant à la philosophe Geneviève Fraisse : "le spectre du consentement va de " adhérer à " jusqu'à " se résigner à " " (Abolition 2012, 2013). Autre organisation pour laquelle le consentement, sous certaines conditions, n'est pas recevable : I'Onu. Cette dernière a en effet publié en 2004, en annexe à la "Convention des Nations Unies contre la criminalité transnationale organisée ", un "Protocole visant à prévenir, réprimer et punir la traite des personnes, en particulier des femmes et des enfants " selon lequel, dans les conditions de la traite, le consentement des personnes qui en sont 
victimes est indifférent : " Le consentement d'une victime de la traite des personnes à l'exploitation envisagée, telle qu'énoncée à I'alinéa "a" du présent article, est indifférent lorsque l'un quelconque des moyens énoncés à l'alinéa "a" a été utilisé ". Ces moyens sont " la menace de recours ou le recours à la force ou à d'autres formes de contrainte, par enlèvement, fraude, tromperie, abus d'autorité ou d'une situation de vulnérabilité, ou par l'offre ou l'acceptation de paiements ou d'avantages pour obtenir le consentement d'une personne ayant autorité sur une autre aux fins d'exploitation » (Onu, 2004).

\section{Des difficultés à juger de la valeur du consentement}

Mais, et si l'on suit Geneviève Fraisse jusqu'au bout de sa réflexion, ce n'est pas un argument pour remettre en cause l'authenticité du consentement. Il est en effet compliqué et périlleux, y compris sur le plan juridique où la notion de consentement est centrale, de chercher à rentrer dans l'intimité de la personne pour juger de la valeur de son consentement. Le propos de la philosophe est plutôt de mettre en lumière les tensions et problématiques entre la dimension intime du consentement énoncé à la première personne du singulier (" je consens ») et le sens politique de celui-ci (Fraisse, 2007).

Une autre philosophe, Michela Marzano, a également insisté sur la complexité du consentement, avec d'un côté l'impossibilité de réduire la personne à ce qu'elle dit, dans la mesure où celle-ci n'étant pas transparente à elle-même et ne jouissant pas d'une liberté absolue et inconditionnelle, il y a aussi à entendre ce qui se dit par-delà ce qui est consciemment dit, attitude qui est la base de toute approche psychothérapeutique ; et d'un autre côté, le fait que la personne $n^{\prime}$ est pas transparente à elle-même, que sa parole peut être emprunte d'ambivalence, et qu'elle s'énonce toujours dans un contexte fait de contraintes et de conditionnements, n'autorise pas à nier sa parole en prétendant savoir pour elle et mieux qu'elle ce qu'il en est de son désir (Marzano, 2006).

\section{Consentement et contraintes}

C'est dans cette logique que semble s'inscrire le protocole de l'Onu. Celui-ci ne tient en effet pas pour indifférent tout consentement, mais tout consentement énoncé dans des circonstances telles que sa prise en compte est jugée non pertinente. Or ces circonstances sont relativement cadrées et recouvrent des éléments généralement retenus juridiquement pour qualifier un viol ou une agression sexuelle. II ne suffit donc pas que le consentement soit exprimé dans un contexte où s'exercent certaines contraintes pour ne pas être pris en compte (sans quoi aucun consentement d'aucun ordre ne pourrait être pris en compte, sauf à imaginer une liberté qui se définirait pas l'absence totale de contraintes, c'est-à-dire une liberté qui ne concernerait pas la condition humaine); il faut que ces 
contraintes soient spécifiques et soient déjà en elles-mêmes condamnables par la loi (enlèvement, fraude, tromperie, abus d'autorité...).

\section{La dignité au-dessus du consentement}

La question est alors de savoir dans quelle mesure une approche non individualiste de la personne peut recourir à des éléments extérieurs à celle-ci pour reconnaître ou condamner des pratiques et activités impliquant des rapports sexuels, même lorsque ceux-ci sont consentis et alors qu'ils ne constituent pas un délit ni un crime. Il ne s'agit pas ici de poser la question sous un angle juridique, mais d'interroger des positions morales qui réprouvent certains rapports sexuels consentis et ne tombant pas sous le coup de la loi. Ainsi en est-il de certains discours féministes concernant le travail sexuel, comme nous l'avons déjà vu, mais aussi concernant le sadomasochisme. À ces contextes de rapports sexuels, est opposée une notion qui transcende les individus : celle de dignité humaine.

\section{L'approche kantienne de la dignité de l'humanité}

La dignité s'entend comme la conformité à un rang considéré comme chargé d'une valeur de référence. Le contraire de la dignité est alors le rabaissement par rapport à cette valeur, une déchéance. La dignité humaine est celle que confère le statut d'être humain, et elle est égale en tout être humain. L'idée de dignité humaine est corrélée à celle de respect, des autres et de soi, en qualité d'être humain. La notion de dignité humaine est régulièrement invoquée dans les débats éthiques et plus spécialement bioéthiques, mais aussi aujourd'hui en éthique de la sexualité. Le philosophe allemand Emmanuel Kant y fait souvent référence, explicitement ou implicitement. Ce philosophe présente la particularité de référer le concept de dignité à la fois à ceux de liberté, d'autonomie et de respect du sujet, et à l'idée d'une transcendance qui dépasserait tout être humain. Il articule ainsi ce qui aujourd'hui se trouve dans un rapport d'opposition sur le plan philosophique mais aussi juridique comme le soulignent les chercheuses en droit public Charlotte Girard et Stéphanie Hennette-Vauchez, avec d'un côté, la dignité considérée comme un attribut du sujet pouvant être opposée à un tiers (droit subjectif), et d'un autre côté, la dignité considérée comme un attribut de l'humanité, qui transcende les personnes et qui peut leur être opposé (droit objectif), et ce même vis-à-vis de ce à quoi elles auraient consenti (Girard et HennetteVauchez,2005).

L'articulation opérée par Kant et qui évite l'opposition des approches subjective et objective est possible parce que le philosophe conçoit la liberté elle-même comme une transcendance par rapport à la condition humaine, à partir d'un dualisme qu'il établit entre, d'une part, une réalité dite empirique, c'est-à-dire perçue par les sens, ce que l'on peut appeler, pour faire vite, la vie 
concrète avec ses contraintes et des motifs de l'action qui sont conditionnés aux circonstances, aux penchants, etc. ; et d'autre part, une réalité dite intelligible ou suprasensible, qui relève de ce que Kant nomme la raison pure, détachée de tout élément empirique et de tout motif conditionnel pour l'action. Ceci permet à la raison pure de déterminer seulement par elle-même la maxime de ses actions, de façon inconditionnelle et libre, conférant ainsi à ses actions une valeur morale absolue.

On retrouve donc là l'idée problématique d'une liberté absolue, qui permettrait de fonder dans le sujet (l'individu) la valeur de son consentement qui serait elle-même absolue. Pour Kant, c'est cette possibilité de la raison de se donner sa propre loi, c'est-à-dire son autonomie et sa liberté, qui constitue sa moralité, et qui fait sa valeur intrinsèque. Tout être doué de raison (tout être humain) est ainsi selon lui une fin en soi, et pas seulement un moyen. Tel est ce que Kant désigne comme étant la dignité de l'humanité (Kant, 1848).

\section{La dignité opposée à la liberté individuelle}

Or non sans raisons valables, cette liberté absolue de la raison n'est pas le point de départ des positions féministes évoquées plus haut. Il s'agit au contraire de partir des conditions concrètes de la vie, et donc de prendre en considération les contraintes qui s'y exercent. La notion de dignité se trouve ainsi devoir référer à autre chose qu'à une liberté absolue des personnes, qui ne peut fonder le consentement in concreto. À ce point, une conception de la liberté comme relative aurait pu être retenue, et le consentement aurait pu être conçu comme lui-même relatif, avec des niveaux de contraintes variables. Lorsque ces contraintes deviennent telles (par exemple celles énoncées par l'Office contre la drogue et le crime de l'Onu), il peut être considéré en effet que le consentement est indifférent, parce qu'il ne peut vraiment s'exercer. Mais les positions féministes sus-citées n'empruntent pas cette voie. II semble en effet que faute de la possibilité d'une liberté absolue, c'est-à-dire d'une transcendance en quelque sorte au cœur de la personne (telle que Kant l'élabore), elles recourent, en particulier concernant le travail sexuel, à une autre transcendance, placée en une conception de la dignité humaine détachée de la volonté des personnes, qui permet de faire fi du consentement de celles-ci, même lorsqu'il est clairement formulé et que le niveau de contraintes qui s'exerce ne relève pas, notamment, du recours à la force.

\section{La dignité humaine transcendante appliquée au travail sexuel}

L'application d'une telle approche à propos du travail sexuel a pour effet (mais parce que c'est aussi ce qui est posé dès le départ) de gommer toute distinction entre le travail sexuel et le viol. Dès lors, toutes les personnes exerçant une activité prostitutionnelle, et ce 
quelles que soient les conditions d'exercice, sont considérées comme des victimes. Plus spécialement, les organisations féministes abolitionnistes ou prohibitionnistes considèrent la prostitution comme une violence faite aux femmes, bien que des hommes également exercent cette activité, pour d'autres hommes comme pour des femmes, dans le cadre du tourisme sexuel notamment pour les femmes clientes (Pruitt et LaFont, 1995 ; Sánchez Taylor, 2001 ; Salomon, 2009).

Il faut bien insister ici sur le fait suivant, essentiel par rapport à la question du consentement : la considération comme victimes des personnes exerçant un travail sexuel ne s'étend pas seulement aux personnes adultes subissant la traite et les réseaux criminels de la prostitution sur fond de misère économique et sociale (personnes originaires de pays du tiers monde, d'Europe de l'Est...), ni aux personnes forcées par un tiers à exercer la prostitution (proxénétisme), ni aux mineurs ; elle s'étend également aux personnes majeures qui ne subissent aucune de ces contraintes (ce qui ne veut pas dire sans aucune contrainte, comme celle de gagner sa vie) et qui affirment exercer un travail sexuel par choix (personnes originaires d'Europe de l'Ouest, d'Amérique du Nord...). $C^{\prime}$ est donc que le principe de non-pertinence du consentement tel qu'il est énoncé dans le protocole de l'Onu se trouve étendu à tout travail sexuel quelles qu'en soient les conditions d'exercice. Ainsi, si la problématique du consentement se trouve, comme l'ont formulé Geneviève Fraisse ou Marchela Marzano, dans l'articulation entre sphère individuelle (privée, intime) et sphère collective (publique, politique), la position de transcendance de la dignité humaine suppose résoudre la tension entre les deux en supprimant la sphère individuelle de l'équation.

\section{Le consentement invalidé par le vécu infantile traumatique ?}

Généralement, la sphère individuelle est invoquée par la position de transcendance de la dignité humaine pour mettre en avant des raisons supposées rendre compte de la formulation du consentement et le disqualifiant. Les associations abolitionnistes affirment en effet que la plupart des personnes exerçant un travail sexuel ont subi des abus sexuels dans leur enfance, souvent de nature incestueuse (ou, à défaut, des carences affectives, ce qui étend le champ des personnes concernées et pose question sur la rigueur de l'argument). Elles s'appuient sur des publications qui promeuvent l'existence d'un lien entre abus sexuels dans l'enfance et travail sexuel concernant les ressortissants d'Europe de l'Ouest ou d'Amérique du Nord, comme celles du sociologue Richard Poulin, qui affirme : « ... ce n'est pas n'importe quelle femme, ni n'importe quel homme qui accepte de faire "volontairement" ce "métier", qui réussit à passer à l'acte, à (sur)vivre dans un autre monde, même si l'argent constitue un attrait et le désir d'échapper aux piètres conditions sociales d'existence, une motivation. S'il y a des causes alimentaires et de survie à la prostitution, il existe aussi un 
déterminisme social et psychologique qui prédispose certaines personnes à la prostitution (. . .). Nombres de recherches vont dans ce sens : les prostitués ont un lourd passé traumatique et une histoire d'abus sexuels chroniques » (Poulin, 2003).

Ces publications et les statistiques qu'elles avancent font l'objet de controverses (Chaumont, 2012 ; Lilian, 2012). Mais la question philosophique en amont de celle concernant la véracité statistique établissant une corrélation entre des abus sexuels subis dans l'enfance, notamment incestueux, et la pratique d'un travail sexuel à l'âge adulte, est de savoir si le fait d'avoir éventuellement vécu des traumatismes sexuels dans l'enfance invalide a priori toute expression de consentement au travail sexuel à l'âge adulte. Cette question est même en réalité plus large et va au-delà du seul champ du travail sexuel, d'une part, et du champ sexuel, d'autre part : le fait d'avoir vécu dans l'enfance des traumatismes, sexuels ou autres, invalide-t-il a priori toute formulation de consentement à l'âge adulte dans ses choix de vie ? II n'y a pas de raisons en effet d'établir à propos du consentement un principe d'exception concernant le travail sexuel, dans la mesure où l'on sait suffisamment, dans les milieux de la psychologie, de la psychanalyse et de la sexologie, que des choix de vie ou des comportements à l'âge adulte sont conditionnés par le vécu dans l'enfance, que ces choix soient sexuels ou autres.

Si l'on répond par l'affirmative à la question - et même si l'on répond ainsi en considérant le champ du travail sexuel comme un domaine d'exception - alors, on retrouve le problème posé par Geneviève Fraisse lorsqu'on veut juger de la valeur du consentement à partir d'une exploration de l'intimité de la personne. Et en ne restreignant pas la question au travail sexuel, c'est la possibilité même de tout consentement, sexuel et autre, qui vacille, dans la mesure où toute personne fait ses choix de vie avec pour déterminant le vécu infantile et ses traumatismes, y compris les féministes abolitionnistes, les sexologues, etc.

\section{Une dignité humaine désubjectivante}

La dignité humaine prend alors un sens qui repose sur la désubjectivation préalable des personnes qui consentent, par leur pathologisation et leur médicalisation, qui procède de surcroît d'un recours constant à la notion d'état de stress post-traumatique dans les discours abolitionnistes pour désigner le travail sexuel comme expérience dévastatrice (David, 2008). Ceci revient à leur refuser de pouvoir endosser la responsabilité de leurs choix, quitte à ce que ces personnes regrettent ces choix ensuite, ou fassent d'autres choix. Le rejet du concept de liberté comme absolue conduit les abolitionnistes à nier toute liberté possible, y compris non absolutiste, c'est-à-dire articulée, même problématiquement, aux notions de contrainte et de conditionnement. La dignité humaine se trouve alors logée à la place laissée vacante par la liberté, et se 
retrouve paradoxalement associée à la non-reconnaissance des êtres humains comme des sujets responsables.

\section{Tensions féministes à propos du sadomasochisme}

Le même genre de problématique se retrouve concernant les pratiques sadomasochistes. Mais bien que les critiques du sadomasochisme par le féminisme radical de la fin du XXe siècle, au départ aux États-Unis, insistent sur la dimension de rabaissement des femmes vis-à-vis des hommes dans les pratiques sadomasochistes et plus globalement dans les rapports sexuels en général comme organisés par la domination masculine (Linden et al., 1982 ; MacKinnon, 1989), on n'y retrouve pas de référence à la dignité humaine. La question est en effet posée sous des angles politiques, sociaux et anthropologiques plutôt que moraux.

\section{Défense du BDSM sous l'angle anthropologique par Gayle Rubin}

Les débats sur le sadomasochisme, généralement associés à la pornographie, furent particulièrement vigoureux au sein même des mouvements féministes. Plusieurs auteurs de l'ouvrage collectif Against Sadomasochism : A Radical Feminist Analysis (Linden et al., 1982) étaient également membres du groupe féministe Women Against Violence in Pornography and Media, contre lequel s'est élevée notamment l'anthropologue Gayle Rubin, qui a débuté sa thèse d'anthropologie sur la communauté cuir et sadomasochiste gay masculine de San Francisco à la fin des années 1970 pour la soutenir en 1994 (Rubin, 1994). Elle a également fondé en 1978 à San Francisco une association luttant pour les droits des lesbiennes sadomasochistes, appelé "Samois ", du nom du personnage fictif de la dominatrice lesbienne Anne-Marie dans Histoire d'O. Cette anthropologue féministe, lesbienne et adepte du BDSM a ainsi pu dénoncer l'approche purement théorique et idéologique de féministes radicales, dénuée de toute dimension empirique : " De nos jours, les textes radicaux (essentiellement féministes) sur le SM sont un ramassis éhonté d'idées fausses et d'informations inexactes, doublées d'un refus obstiné d'accepter ce qui viendrait contredire les idées toutes faites " (Rubin, 2010).

\section{Dignité humaine et sadomasochisme : une approche juridique}

Ce n'est qu'à la toute fin du XXe siècle, et par l'entremise de réflexions juridiques, que le principe de dignité humaine sera opposé aux pratiques sadomasochistes. À ce titre, une affaire à propos de pratiques sadomasochistes particulièrement violentes, portée en appel en 2005 devant la Cour européenne des Droits de I'homme (CEDH), fait référence et polémique (affaire K. A. et A. D. contre Belgique). La première section de la CEDH a en effet considéré que " la faculté pour chacun de mener sa vie comme il l'entend peut également inclure la possibilité de s'adonner à des activités perçues comme étant d'une nature physiquement ou 
moralement dommageable ou dangereuse pour sa personne " (CEDH, 2005). Or la juriste Muriel Fabre-Magnan a considéré que cet arrêt renie la primauté du concept de dignité humaine sur celui d'autonomie de la personne (Fabre-Magnan, 2006). Elle a dénoncé par ailleurs que le consentement soit « un sésame de nature à lever toutes les interdictions et à légitimer tous les comportements, signe juridique moderne de l'individualisme et du libéralisme " (FabreMagnan, 2007a). Même en cas de consentement, selon elle, " nul ne peut valablement consentir à ce que lui soient portées des atteintes contraires à la dignité de la personne humaine et donc à renoncer à cette dignité » (Fabre-Magnan, 2007b). Là encore, donc, la dignité humaine fonctionne comme un principe transcendant, et se rapproche même de sa première signification hiérarchique de "dignitas" (rang social ou d'une fonction), qui impose un devoir plus qu'elle n'ouvre à des droits.

\section{Conclusion}

La question du consentement (ici sexuel, mais elle se pose de la même manière d'une façon plus générale) se trouve ainsi posée à un double niveau : sur quoi le faire reposer, d'une part (désir ou volonté), et d'autre part, dans quelle mesure doit-on en tenir compte et à partir de quand peut-on éventuellement le considérer comme indifférent. L'opposition des débats s'articule entre ce qui serait, d'un côté, une représentation individualiste de la liberté (désignée comme libérale sous nos latitudes), et d'un autre côté, une représentation collective et plus politique, sinon morale, de l'être humain. Cette dichotomie ne saurait cependant résumer les positionnements possibles sur les questions du consentement et de la dignité humaine. En donnant la primauté à l'individu sur le collectif quant à ce qui constitue l'être même de l'individu (comme Descartes) ou sur le plan comportemental (comme la théorie de I'«homo oeconomicus » dans le modèle néo-classique en économie), l'individualisme fait passer au second plan la condition fondamentalement sociale de l'être humain. Poussé au paroxysme de sa logique, l'individualisme est une métaphysique de l'individu, en ce qu'il érige un individu antérieur au monde social, dans lequel il est placé secondairement, et où il agit en fonction de ses supposées propres lois internes, en référence à une nature humaine individuelle (Mill, 2006). Or il y a bien quelque chose qui dépasse et précède chaque individu pris isolément, à savoir la réalité sociale, qui n'est pas la seule somme des individus qui la compose, mais l'environnement dans et par lequel les individus deviennent ce qu'ils deviennent. Mais la notion de dignité humaine inspirée de Kant réintroduit une forme de transcendance de type religieux ou métaphysique, ce qui pose aussi bien un problème philosophique que politique, dans des démocraties non seulement soucieuses des libertés individuelles, mais également laïques. Le consentement doit alors pouvoir être pensé comme articulant la liberté individuelle à des principes collectifs, l'une et les autres situés sur un plan 
d'immanence à la réalité humaine, et non à partir de positions métaphysiques.

\section{References}

Abolition 2012. Prostitution = violence ! Dossier de presse; 2013http://www.abolition2012.fr/DPprostitution-violence1.pdf

Bajos N, Bozon M. (eds.) Enquête sur la sexualité en France : pratiques, genre et santé (eds.). Paris: La Découverte; 2008.

Bajos N, Giami A, Laurent R, Leridon H, Spira A. Comportements sexuels et sida en France. Données de l'enquête Analyse des comportements sexuels en France. Paris: éditions Inserm;1998.

Black MC, Basile KC, Breiding MJ, SmithMikel SG, Walters L, MerrickMT, et al. The National Intimate Partner and Sexual Violence Survey: 2010 Summary Report. Atlanta: National Center for Injury Prevention and Control; 2011.

Caouette A-A.Lalonde C, editor. "Vendre son oui ». Montréal: Le Devoir; 2015. http://www.ledevoir.com/societe/actualites-ensociete/433574/vendre-son-oui

CEDH. CEDH première section. Arrêt 17 février 2005; 2005 http://hudoc.echr.coe.int/sites/fra/pages/search.aspx?i=001-68354

Charpenel Y. Rapport mondial sur l'exploitation sexuelle. Paris: Economica; 2012.

Chaumont JM. Le militant, l'idéologue et le chercheur. Debat 2012; 172:120-30.

David M. Santé mentale et usage idéologique de l'« état de stress post-traumatique » dans les discours sur la prostitution et la traite. Rech Sociol Anthropol 2008:39-41, 55-70.

Descartes R. Discours de la méthode (1637). Paris: Gallimard, La Pléiade; 1953a.

Descartes R. Les passions de l'âme (1649). Paris: Gallimard, La Pléiade; 1953c.

Fabre-Magnan M. Le sadomasochisme n'est pas un droit de I'homme. Conference 2006;22:265-96.

Fabre-Magnan M. La dignité en Droit : un axiome. Rev Interdiscip Etud Jurid 2007a;58:1-30.

Fabre-Magnan M. Dignité : dignité et respect du corps. In: Marzano M, editor. Dictionnaire du corps. Paris: PUF; 2007b. p. 307-13. 
Fraisse G. Du consentement. Paris: Seuil; 2007.

Freud S. Métapsychologie (1915). Paris: Gallimard; 1968.

Girard C, Hennette-Vauchez S. La dignité de la personne humaine : recherche sur un processus de juridicisation. Paris: PUF; 2005.

Kant E. Fondements de la métaphysique des mœurs. In: Critique de la Raison pratique précédé des Fondements de la métaphysique des mœurs. Paris: Librairie philosophique de Ladrange; 1848. p.3-126.

Lilian M. De l'objectivation à l'émotion. La mobilisation des chiffres dans le mouvement abolitionniste contemporain. Mots 2012;100:173-85.

Linden F, Pagano D, Russell D, Star S. Against sadomasochism: a radical feminist analysis. Palo Alto: Frog in the Well; 1982.

MacKinnon C. Toward a Feminist Theory of the State. Cambridge: Harvard University Press; 1989.

Martine J. Le viol-location. Liberté sexuelle et prostitution. Paris: L'Harmattan; 2013.

Marzano M. Je consens, donc je suis. Paris: PUF; 2006.

Mill JS. La psychologie et les sciences morales : sixième chapitre du système de logique (1843). Paris: L'Harmattan; 2006.

Onu. Office contre la drogue et le crime. Protocole visant à prévenir, réprimer et punir la traite des personnes, en particulier des femmes et des enfants, additionnel à la Convention des Nations Unies contre la criminalité transnationale organisée. New York: ONU; 2004.

OLF. Osez le féminisme ! (OLF). Pourquoi est-il nécessaire de réaffirmer la position abolitionniste de la France en matière de prostitution; 2011

http://www.osezlefeminisme.fr/sites/www.osezlefeminisme.fr/files /documents/Dossier\%20d\%C3 \%A9put\%C3 \%A9s\%20r\%C3 \%A9solution\%20abolition\%20prostitution\%20-\%200LF.pdf

Olivier M. Rapport d'information fait au nom de la délégation aux droits des femmes et à l'égalité des chances entre les hommes et les femmes sur le renforcement de la lutte contre le système prostitutionnel. Paris: Assemblée Nationale; 2013. p. 1360.

Organisation mondiale de la santé (OMS). La violence à l'encontre des femmes : violence d'un partenaire intime et violence sexuelle à l'encontre des femmes. Aide-mémoire; 2014. p.

239,http://www.who.int/mediacentre/factsheets/fs239/fr/ 
Poulin R. Prostitution, crime organisé et marchandisation. TiersMonde 2003; 44:735-70.

Pruitt D, LaFont S. For love and money: Romance Tourism inJamaica. Ann Tour Res 1995; 22:422-40.

Richters J, de Visser RO, Rissel CE, Grulich AE, Smith AMA. Demographic and psychosocial features of participants in bondage and discipline, "sadomasochism » or dominance and submission (BDSM): data from a national survey. J Sex Med 2008; 5:1660-8.

Rubin G. The valley of the kings: leathermen in San Francisco,19601990. University of Michigan; 1994, PhD diss.

Rubin G. Le péril cuir: remarques sur la politique et le SM. In: Surveiller et jouir : anthropologie politique du sexe. Paris: Epel; 2010. p. 83-134.

Salomon C. Vers le Nord. Autre part 2009; 49:223-40.

Sánchez Taylor J. Dollars are a girl's best friend? Female tourists'sexual behaviour in the Caribbean. Sociology 2001;35:74964.

Schaffauser T. Prostitution. Chiffres biaisés, amalgames : les mensonges de la proposition de loi. L'Obs-Le Plus; 2014 http://leplus.nouvelobs.com/contribution/1192812-prostitutionchiffres-biaises-amalgames-les-mensonges-de-la-proposition-deloi.html

Simard D. Affaire DSK une prostituée serait-elle inviolable ? Rue 89;2011 http://rue89.nouvelobs.com/2011/07/07/affaire-dsk-uneprostituee-serait-elle-inviolable-212489

Spinoza B. Traité théologico-politique (1670). H. Diaz; 1842.Spinoza B. Éthique (1677). Paris: Garnier-Frères; 1965.

Weitzer R. Flawed theory and method in studies of prostitution. Violence Against Women 2005; 11:934-49. 